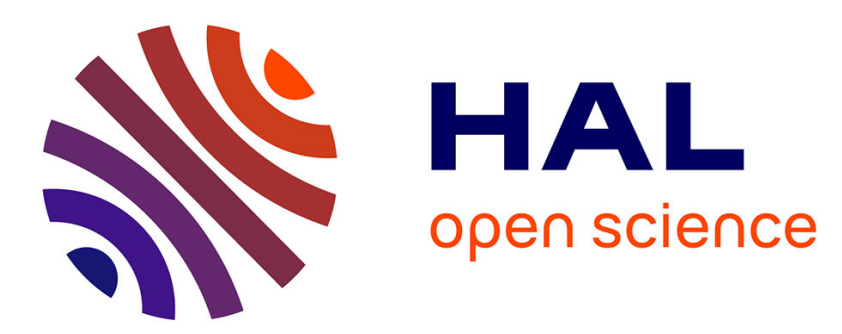

\title{
Habitat et pratiques domestiques des Ve-IVe s. av. J.-C. dans la ville de Lattes \\ Maria-Carmen Belarte
}

\section{To cite this version:}

Maria-Carmen Belarte. Habitat et pratiques domestiques des Ve-IVe s. av. J.-C. dans la ville de Lattes. Gallia - Archéologie de la France antique, 2008, Lattara/Lattes (Hérault): nouveaux acquis, nouvelles questions sur une ville portuaire protohistorique et romaine, 65, pp.91-106. 10.3406/galia.2008.3333 . hal-01913791

\section{HAL Id: hal-01913791 \\ https://hal.science/hal-01913791}

Submitted on 19 Dec 2019

HAL is a multi-disciplinary open access archive for the deposit and dissemination of scientific research documents, whether they are published or not. The documents may come from teaching and research institutions in France or abroad, or from public or private research centers.
L'archive ouverte pluridisciplinaire HAL, est destinée au dépôt et à la diffusion de documents scientifiques de niveau recherche, publiés ou non, émanant des établissements d'enseignement et de recherche français ou étrangers, des laboratoires publics ou privés.

\section{(ㅇ)(1) $\$$}

Distributed under a Creative Commons Attribution - NonCommercial - NoDerivatives $\mid 4.0$ 


\title{
HABITAT ET PRATIQUES DOMESTIQUES DES V $V^{\mathrm{e}}-\mathrm{IV}^{\mathrm{e}} \mathrm{S}$. AV. J.-C. DANS LA VILLE DE LATTES
}

\author{
Maria-Carmen BELARTE
}

Mots-clés. Âge du Fer, architecture, techniques de construction, urbanisme, espaces domestiques, aménagements domestiques.

Résumé. Les recherches menées ces dernières années sur le site de Lattes ont livré une documentation abondante sur l'architecture et l'organisation de l'espace domestique $d u V^{e}$ s. et surtout du $I V^{e} s$. av. J.-C. C'est en effet durant cette période qu'a eu lieu la mise en place de la trame urbaine de la ville qui, dans ses grandes lignes, perdurera jusqu'à la fin de l'occupation protohistorique du site; de même, c'est à ce moment qu'apparaissent des techniques de construction dont certaines sont exclusives de ces périodes, mais qui pour d'autres resteront en vigueur jusqu'à la Protohistoire récente. Cet article présente une synthèse de nos connaissances sur cette phase ancienne, en insistant sur les permanences, les modifications ou les évolutions dans l'architecture, la typologie des maisons et les pratiques domestiques au cours de ces étapes et par rapport aux étapes postérieures.

Key-words. Iron Age, architecture, building techniques, urbanism, private space, household equipment.

Abstract. During the last years research on Lattes, a rich documentation has been recorded about the architecture and organization of private space during the $5^{\text {th }}$ century B.C. and especially the $4^{\text {th }}$ century. During this link of time actually, the urban grid system is being laid and it will last until the end of the Protohistoric occupation of the site. Building techniques also appear, some of which are exclusive of this period but some others will last until the Late Protohistory. This paper sums up our knowledge of this early phase, putting the point on the continuity, change or evolution involved in architecture, dwelling typology and household practices along these phases in comparison with following phases.

Translation: Isabelle FAUDUET

Schlüsselwörter. Eisenzeit, Architektur, Konstruktionstechniken, Urbanismus, häusliche Bereiche, häusliche Einrichtungen.

Zusammenfassung. Die Untersuchungen der letzten Jahre haben in Lattes eine umfangreiche Dokumentation über die Architektur und die Organisation des häuslichen Bereichs im 5. und vor allem im 4. Jh. v.u.Z. geliefert. In dieser Zeit entsteht nämlich die in ihren großen Linien bis ans Ende der frühgeschichtlichen Besiedlung des Ortes beibehaltene Grundform der Stadt; ebenso bilden sich in dieser Zeit Konstruktionstechniken heraus, von denen einige dieser Zeit eigen sind, andere jedoch bis ans Ende der jüngeren Eisenzeit fortbestehen. Dieser Artikel präsentiert eine Synthese unserer Kenntnisse dieser ersten Phase und setzt dabei den Akzent auf die Kontinuitäten, die Modifikationen und die Evolutionen in der Architektur, die Typologie der Häuser und die häuslichen Gepflogenheiten während dieser und im Vergleich zu den späteren Phasen.

Übersetzung: Isa ODENHARDT-DONVEZ

\section{L'ÉTAT DES CONNAISSANCES}

Les recherches menées ces dernières années sur le site de Lattes ont livré une documentation abondante sur l'architecture et l'organisation de l'espace domestique du $\mathrm{V}^{\mathrm{e}} \mathrm{s}$. et surtout du $\mathrm{IV}^{\mathrm{e}}$ s. av. J.-C. Même si nos connaissances sont encore partielles, nous pouvons à l'heure actuelle restituer quelques aspects de la vie quotidienne et formuler quelques hypothèses sur la trame urbaine et la structure de l'habitat de ces périodes. 


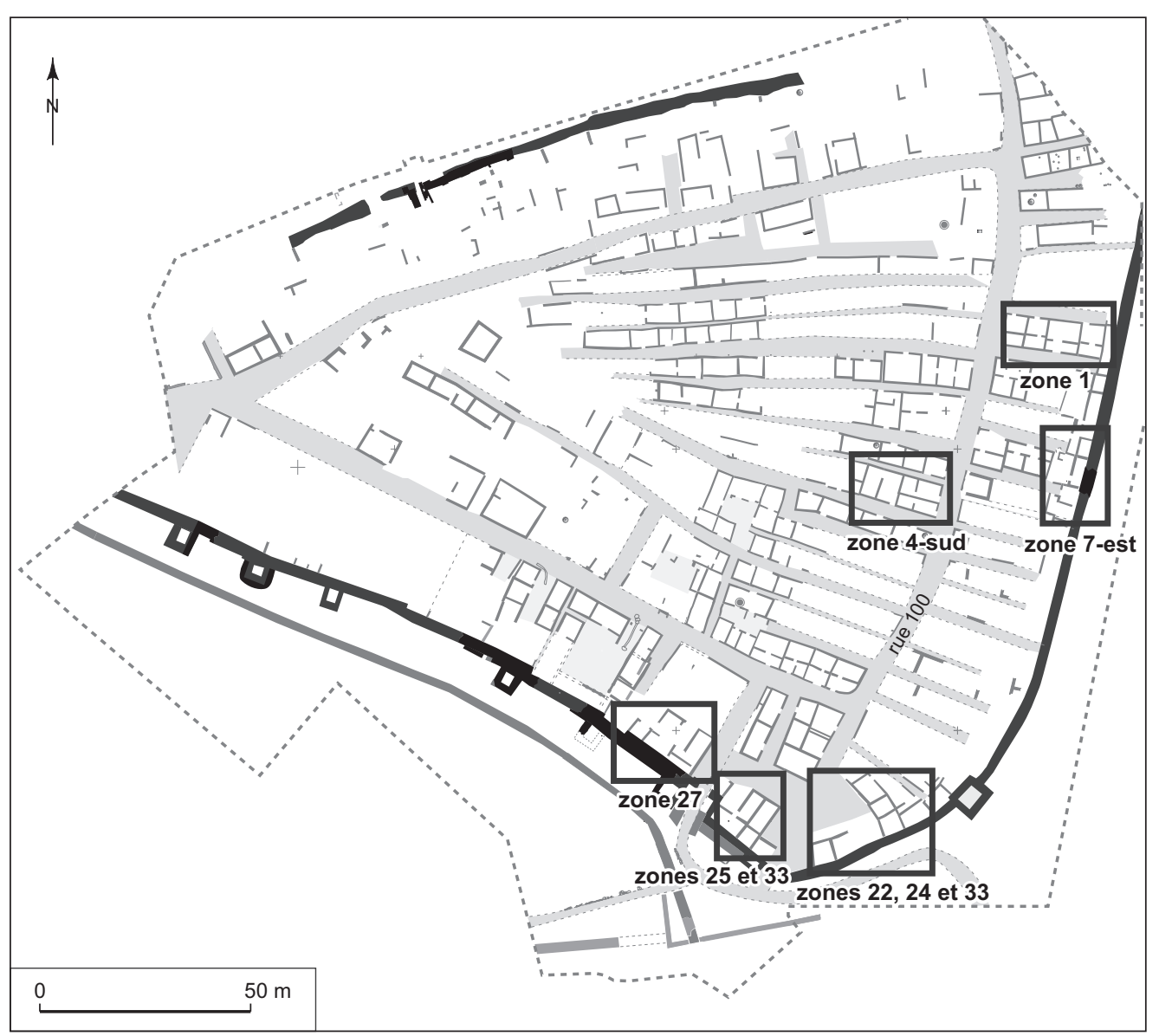

Fig. 63 - Plan général du site de Lattara avec localisation des quartiers mentionnés dans le texte (DAO : (C UFRAL).

Les informations sur le $\mathrm{V}^{\mathrm{e}} \mathrm{s}$. à Lattara proviennent de deux sondages qui ont eu pour but l'étude de l'évolution stratigraphique de deux quartiers d'habitation depuis l'origine de la ville. Le premier de ces sondages est la zone 1, au nord-est du site (fig. 63), entre la façade orientale du rempart et un axe de circulation nord-sud (rue principale 100). Cette zone couvre un rectangle d'une longueur de 26,30 m sur une largeur variant entre 7,50 m et $10,30 \mathrm{~m}$. Le deuxième sondage prend place dans la partie sud de la ville, le long du rempart, en contact direct avec le port par une, puis deux portes ménagées dans l'enceinte ; il correspond à la zone 27 , qui couvre un rectangle de $22 \mathrm{~m}$ sur $10 \mathrm{~m}$.

En ce qui concerne l'habitat du $\mathrm{IV}^{\mathrm{e}}$ s. av. J.-C., nous disposons en outre des données provenant des zones 4 -sud et 7-est et, bien que plus partielles, des observations effectuées dans les zones 22, 24, 25, 32 et 33. Il s'agit dans presque tous les cas de quartiers d'habitat qui se trouvent à la périphérie orientale du site, à proximité du rempart, souvent accolés à lui (fig. 63).

Les résultats disponibles sur l'habitat du $\mathrm{IV}^{\mathrm{e}} \mathrm{s}$. av. J.-C. ont été en grande partie publiés dans le $\mathrm{n}^{\circ} 12$ de la série Lattara (Roux, 1999 ; Lebeaupin, 1999 ; Py, 1999a), ainsi que dans des articles antérieurs (Py, 1996a ; Lebeaupin, 1994 ; López, 1994) ; les données sur le $\mathrm{V}^{\mathrm{e}}$ s. sont, quant à elles, encore inédites. Le but de cet article est donc de présenter une synthèse de nos connaissances sur cette phase ancienne, en insistant sur les permanences, les modifications ou les évolutions dans l'architecture et les pratiques domestiques au cours de ces étapes et par rapport aux étapes postérieures. C'est en effet durant cette période qu'a eu lieu la mise en place de la trame urbaine de la ville qui, dans ses grandes lignes, perdurera jusqu'à la fin de l'occupation protohistorique du site ; de même, c'est à ce moment qu'apparaissent des techniques de construction dont certaines sont exclusives de ces périodes, mais qui 
pour d'autres resteront en vigueur jusqu'à la Protohistoire récente.

\section{LES MAISONS ET LA TRAME URBAINE}

La première occupation protohistorique du site clairement documentée, datée de la fin $\mathrm{du} \mathrm{VI}^{\mathrm{e}} \mathrm{s}$. et du début du $\mathrm{V}^{\mathrm{e}}$ s. av. J.-C., n'est attestée pour l'instant que dans la zone 27. Il s'agit d'un quartier d'habitation dont les maisons, aux murs de terre sur solins en pierre, sont disposées perpendiculairement au rempart; les murs ne s'accolent pas à celui-ci mais en sont séparés par une sorte de couloir de 1,30 m de large. Cet ensemble de maisons, dont le mobilier est presque dans sa totalité de production étrusque, est violemment détruit par un incendie au début du $\mathrm{V}^{\mathrm{e}} \mathrm{s}$. av. J.-C. Nous n'entrerons pas dans le détail de cette occupation dont la problématique est très particulière et qui fait l'objet d'une autre contribution dans ce dossier (voir Lebeaupin, Séjalon, supra, p. 45-64).

Dans le deuxième quart du $\mathrm{V}^{\mathrm{e}} \mathrm{s}$. av. J.-C., le quartier de la zone 27 est reconstruit. On assiste alors à une succession d'occupations de durées diverses, qui présentent aussi des traits différents entre elles : des niveaux où les maisons sont bâties à base de matériaux périssables sur poteaux porteurs et organisées de manière lâche alternent avec des niveaux où les maisons, bâties en brique sur solins de pierre, sont appuyées au rempart et précédées de cours. Ces différentes occupations sont séparées par des couches de destruction suivies normalement de remblais sur lesquels s'installeront de nouvelles maisons, parfois précédées par des bâtiments de très courte durée. De tels éléments témoignent d'une phase où il n'existe pas encore une structuration stable de l'espace, et dans laquelle la trame urbaine s'organise petit à petit, pour être pleinement fixée à la fin $d u \mathrm{~V}^{\mathrm{e}} \mathrm{s}$.

Les données obtenues sur le $\mathrm{V}^{\mathrm{e}} \mathrm{s}$. dans la zone 1 témoignent d'une évolution semblable. Pour ce quartier, on ne connaît pas encore les niveaux correspondant à la fondation du site ; cependant les fouilles menées entre 2001 et 2006 ont livré une abondante documentation sur la seconde moitié $d u \mathrm{~V}^{\mathrm{e}}$ s. Dans ce cas, on assiste également à une alternance entre des îlots formés par des maisons accolées ou parallèles au rempart, parfois séparées par des venelles, et des phases d'occupation où l'urbanisme se présente de manière plus lâche, avec des espaces ouverts, des bâtiments en matériaux périssables, etc. Tout se passe comme s'il s'agissait d'une phase de mise en place de l'urbanisme, où la distribution de l'espace ne semble pas suivre de normes strictes et où la disposition des maisons et des îlots subit des modifications légères lors de chaque réaménagement. Vers le milieu du $\mathrm{V}^{\mathrm{e}} \mathrm{s}$., l'îlot 1 est composé de quatre maisons séparées par une ruelle centrale : de chaque côté de celle-ci, deux maisons sont disposées avec des murs accolés et non mitoyens (fig. 64a et fig. 65). Entre 450 et 425 av. J.-C., plusieurs états se succèdent, durant lesquels les maisons sont séparées par des espaces ouverts (fig. 64b). Entre 425 et 400 av. J.-C., le quartier évolue vers un schéma à quatre maisons séparées par des venelles (fig. 64c) qui préfigure l'organisation du quartier à base de maisons mitoyennes tel qu'il se stabilisera au IV ${ }^{\mathrm{e}} \mathrm{s}$.

Malgré cette diversité des évolutions internes de chaque quartier, il apparaît évident, aussi bien dans la zone 1 que dans la zone 27, que dès 425 av. J.-C. l'urbanisme est déjà organisé en îlots accolés à la fortification ou perpendiculaires à celle-ci.

\section{UNE GRANDE DIVERSITÉ DES TECHNIQUES DE CONSTRUCTION}

Pendant toute l'occupation de la ville, la terre reste le matériau le plus largement employé dans la construction. C'est la conséquence logique de l'emplacement du site dans un milieu lagunaire, où ce matériau est très abondant sur place. La pierre, en revanche, est absente à proximité, ce qui a engendré au cours de l'occupation du site un épierrement constant des murs des bâtiments antérieurs pour remployer ces éléments en quelque sorte précieux. C'est la raison pour laquelle les structures bâties en pierre sont souvent mal conservées.

Le trait principal des périodes anciennes de l'habitat de Lattes est la diversité des modes de construction utilisés, qui peuvent coexister dans un même quartier, dans une même phase, parfois dans une même maison ou dans une même structure.

\section{LES MURS EN TORCHIS ET LES CONSTRUCTIONS EN MATÉRIAUX PÉRISSABLES}

Le premier trait à signaler est la coexistence des constructions à poteaux porteurs, souvent appelées « cabanes ", et de celles à murs porteurs. Durant le $\mathrm{V}^{\mathrm{e}} \mathrm{s}$. av. J.-C., les bâtiments dont les parois sont formées à l'aide d'une armature de clayonnage enduite de torchis sont relativement fréquents (fig. 66). Plusieurs de ces structures ont été identifiées à partir de la présence de tranchées d'ancrage des parois, parfois comblées par des cendres provenant de la crémation 


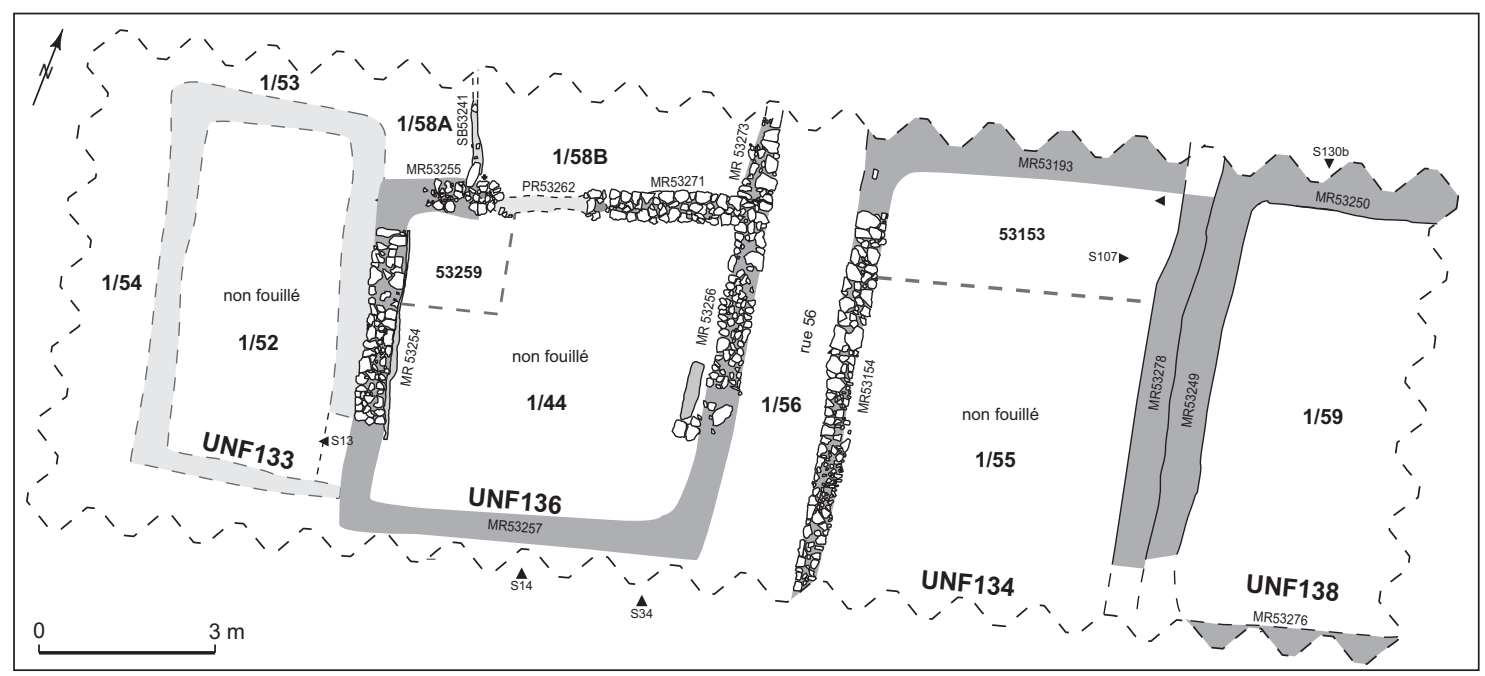

a. Vers 450 av. J.-C.

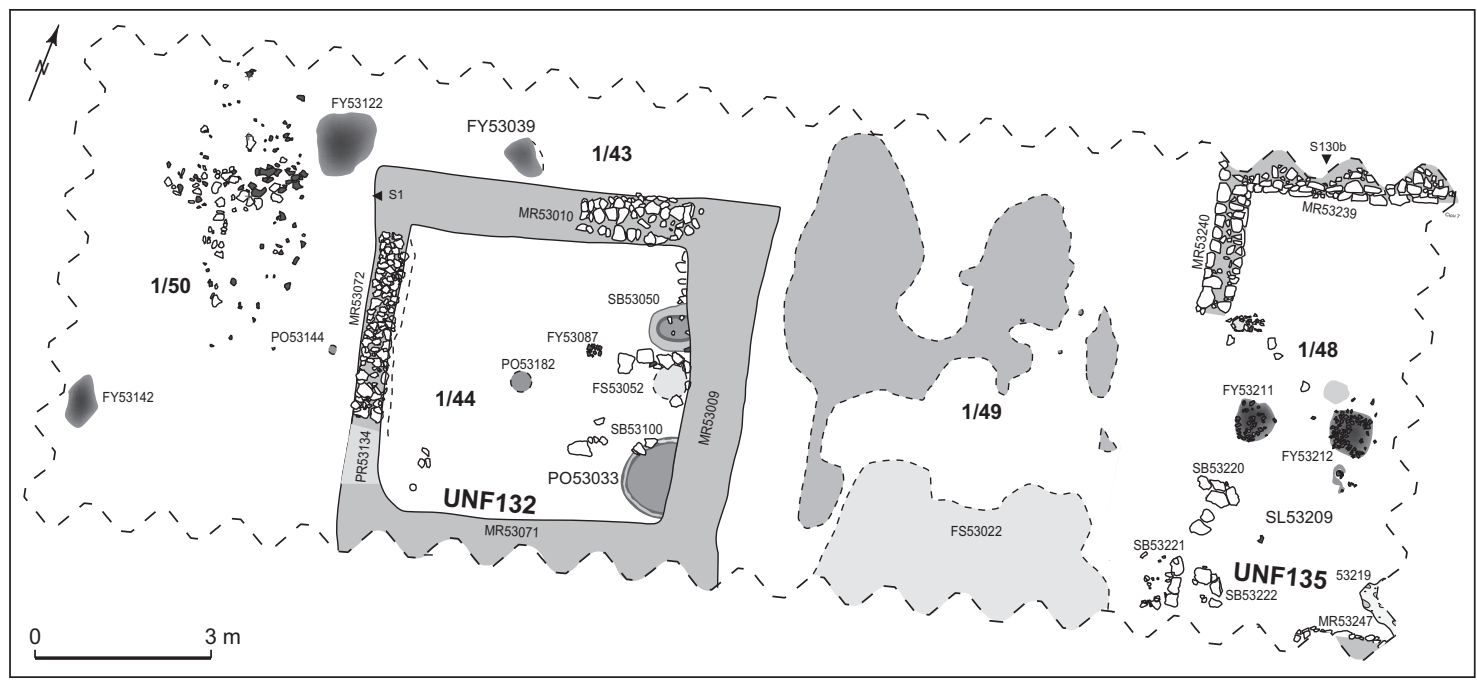

b. Vers $450-425$ av. J.-C.

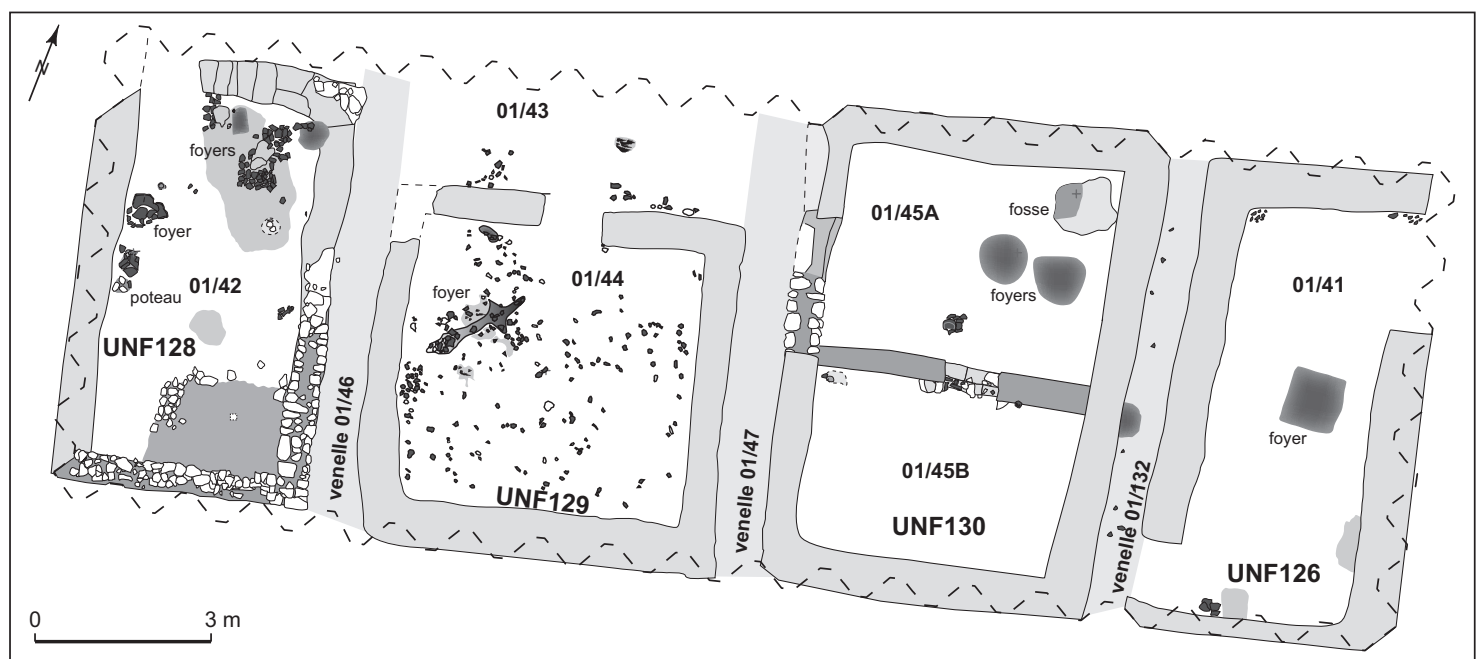

c. Vers 425-400 av. J.-C.

Fig. 64 - Évolution de la zone 1 de Lattes entre 450 et 400 av. J.-C. (DAO : () UFRAL). 


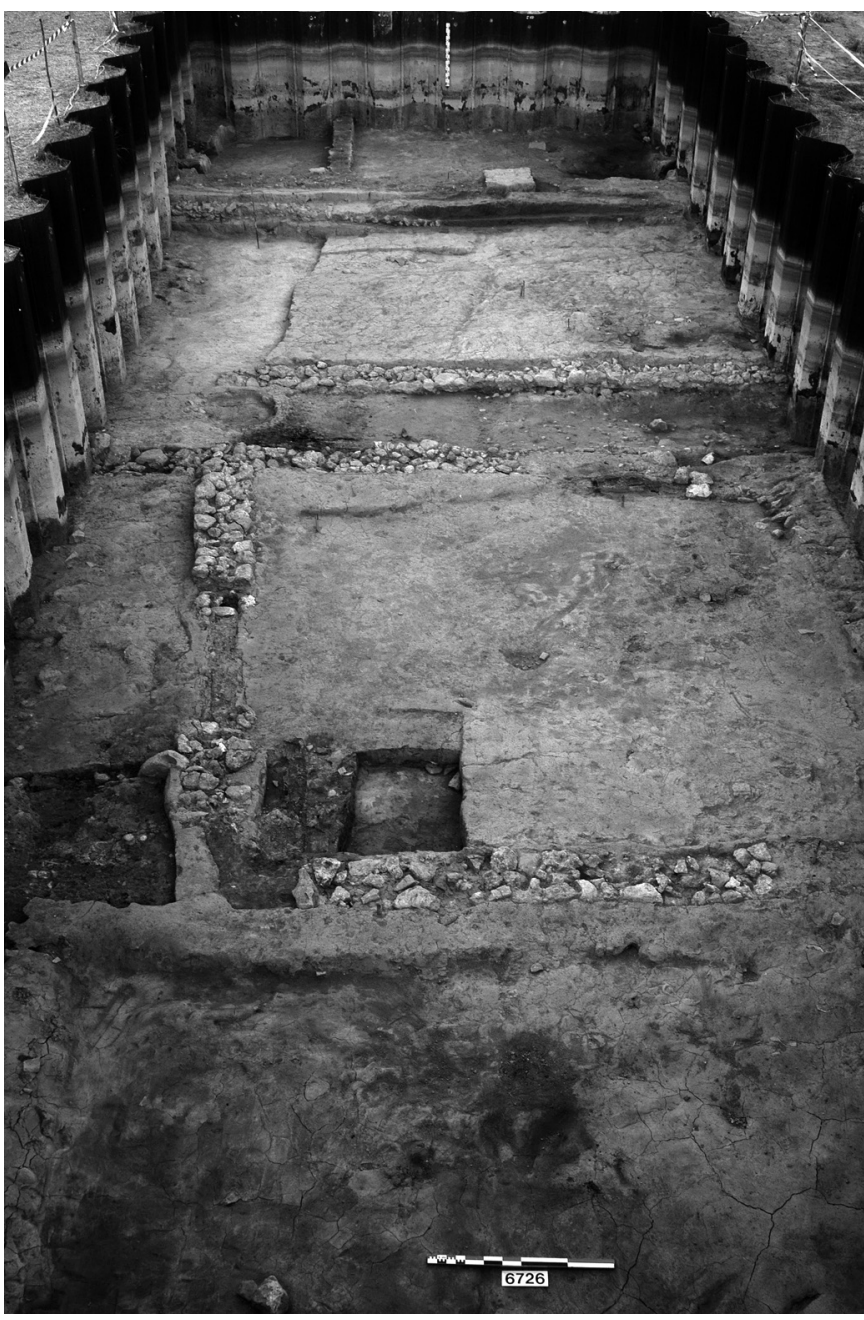

Fig. 65 - Maisons juxtaposées séparées par une ruelle au milieu du $V^{e}$ s. av. J.-C. dans la zone 1. Vue prise de l'ouest (photo : (C) UFRAL).

des végétaux utilisés dans l'élévation, ainsi que des calages de poteaux. Ces bâtis perdurent durant le $\mathrm{IV}^{\mathrm{e}} \mathrm{s}$. et peuvent avoir des fonctions diverses :

- des maisons ou des cabanes correspondant à des occupations temporaires, de courte durée, normalement durant une phase de réaménagement entre deux occupations ;

- des structures annexes à des constructions à murs porteurs : c'est le cas, par exemple, des maisons de la phase F3 de la zone 27, autour de 400 av. J.-C., formées par des pièces de fond à murs en adobe sur solins de pierre, accolées au rempart, précédées par des cours fermées à base de parois en matériaux périssables (fig. 67) ;

- des structures destinées à délimiter des aires spécifiques dans une cour ou une aire extérieure. C'est le cas, par exemple, de la cour partagée par les maisons 123 et 124 de

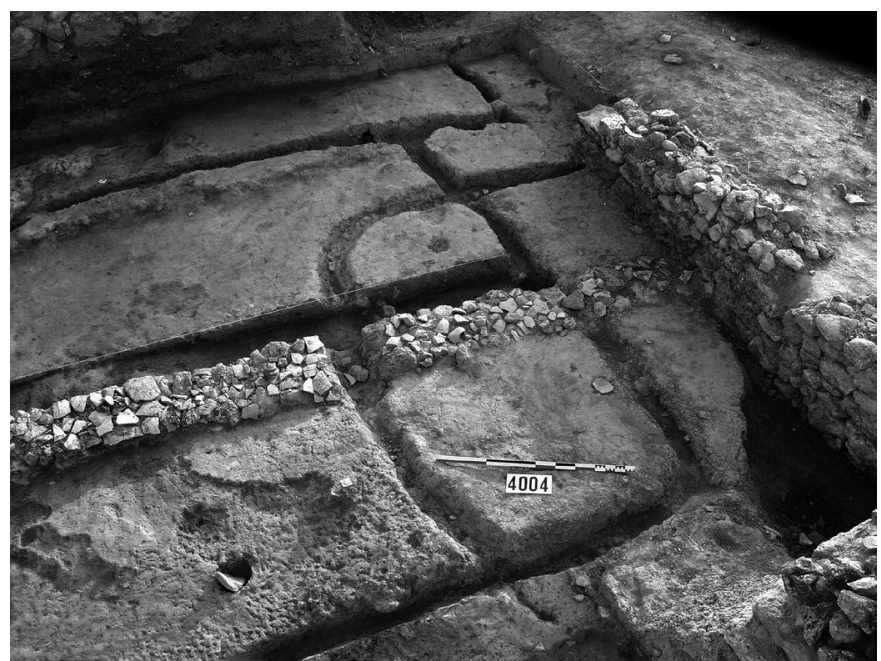

Fig. 66 - Succession d'habitations à murs porteurs et de bâtiments sur poteaux à parois ancrées dans des tranchées dans la zone 27 au troisième quart du $V^{e}$ s. av. J.-C. (photo : (C) UFRAL).

la zone 1 (vers 400 av. J.-C.), ou de la délimitation des aires de foyers ou de fours, qui souvent sont protégées par des couvertes légères soutenues par des parois en torchis ;

- des maisons d'habitation dont les murs sont complètement bâtis à base de végétaux enduits de terre. C'est le cas de la maison 104 de la zone 1 (vers 375 av. J.-C.) (Roux, Chabal, 1996). Les maisons en torchis sont un élément déjà résiduel au $\mathrm{IV}^{\mathrm{e}} \mathrm{s}$., qui aura complètement disparu à la fin de ce siècle.

\section{LES CONSTRUCTIONS SUR MURS PORTEURS EN PIERRE ET EN TERRE}

Les constructions bâties sur murs porteurs deviennent prédominantes à la fin du $\mathrm{V}^{\mathrm{e}} \mathrm{s}$. av. J.-C. Sous ce concept général de "murs porteurs ", on inclut les murs en pierre, en terre ou ceux combinant les deux matériaux. Du point de vue technique, plusieurs combinaisons sont utilisées : des murs en adobe ou en terre massive sur solins en pierre, des murs entièrement en terre massive, des murs en adobe sur solins en terre massive et, très rarement, des murs complètement bâtis en pierre (voir Roux, infra, p. 107-109). La combinaison de matériaux divers existe également dans la dimension horizontale : les murs en terre massive peuvent contenir des parties en pierre, comme dans le cas des murs MR51009 et MR50402 de la zone 1 vers 425 av. J.-C. (fig. 68), bien que cette technique mixte soit surtout employée dans les angles des murs, où la pierre fait fonction de renfort, comme dans l'angle nord-ouest de la maison 3201 (400-375 av. J.-C.) (Py, 1999a, p. 187). 


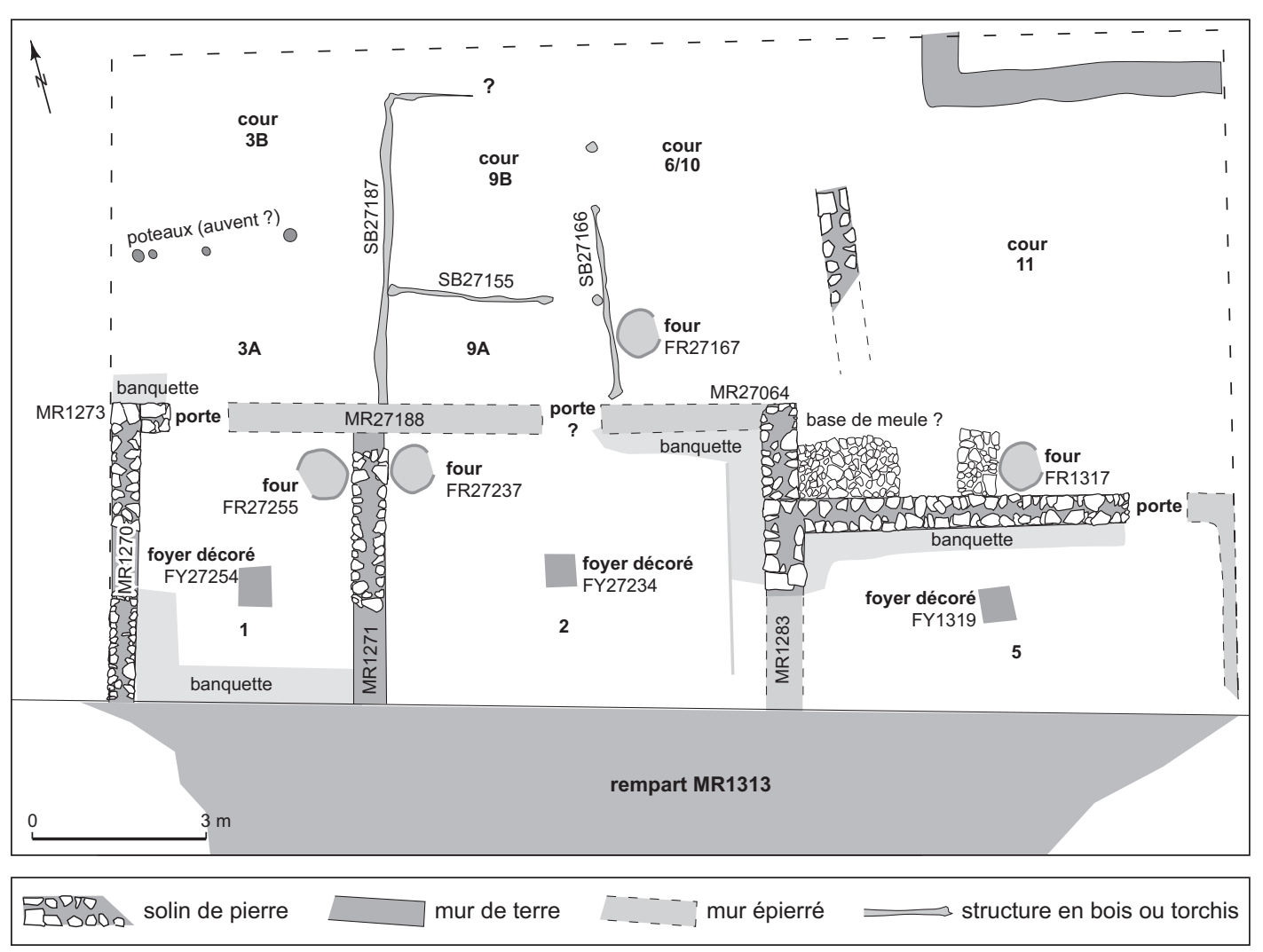

Fig. 67 - Plan de la zone 27 vers 400 av. J.-C., où les maisons à murs porteurs possèdent des avant-cours à parois en matériaux périssables (DAO : (C) UFRAL).

Les murs sont rarement bâtis dans des tranchées de fondation ; le plus souvent, ils sont construits sur des murs antérieurs qui font office de fondations. Cette pratique engendre encore plus de possibilités dans la combinaison des matériaux (par exemple, des solins de pierre bâtis sur des murs en terre massive antérieurs).

\section{LES SOLINS ET MURS EN PIERRE}

Plus ou moins bien conservés, les solins ou soubassements en pierre sous les élévations en terre sont attestés depuis le $\mathrm{V}^{\mathrm{e}} \mathrm{s}$. av. J.-C. Y prédomine la pierre tendre, grossièrement équarrie, avec des éléments de dimensions et de formes diverses (surtout des moellons et des cailloux de $20-25 \mathrm{~cm} \times 15-20 \mathrm{~cm} \times 10-13 \mathrm{~cm}$ en moyenne, plus rarement des blocs), non sélectionnés, disposés en boutisse et panneresse, toujours liés avec de la terre.

Dans quelques cas, les assisses de base des soubassements sont faites de blocs de dimensions plus grandes, qui débordent de quelques centimètres par rapport au parement du mur. On peut prendre comme exemple le mur
MR53240 de la maison 135 de la zone 1 (450-425 av. J.-C.) dont l'assise de base est faite de gros blocs (dimensions moyennes : $34-39 \mathrm{~cm}$ x 28-32 cm x 10-12 cm) disposés en double parement et en panneresse en compagnie de quelques moellons $(20-25 \mathrm{~cm}$ de côté) et cailloux $(10 \mathrm{~cm})$ de forme arrondie.

Ces solins possèdent normalement entre 3 et 4 assises de pierre, dont les hauteurs sont variables; les largeurs sont également très diverses, la majorité étant comprise entre $50 \mathrm{~cm}$ et $80 \mathrm{~cm}$. Très rarement, quelques solins possèdent des hauteurs suffisamment importantes pour supposer que les murs aient été complètement bâtis avec ce matériau, sans élévation de terre. C'est le cas des murs MR50019 et MR50048 de la maison 121 de la zone 1 (400-375 av. J.-C.) dont les élévations en pierre, très homogènes, sont conservées sur plus de $0,90 \mathrm{~m}$ de haut.

L'arase des solins de pierre est souvent aménagée par un placage de tessons qui rend plus facile l'accrochement des élévations en terre. Cette technique sera caractéristique de l'architecture lattoise jusqu'au début du $\mathrm{II}^{\mathrm{e}} \mathrm{s}$. av. J.-C. (Chazelles, 1996, p. 271-272). De même, certains solins 


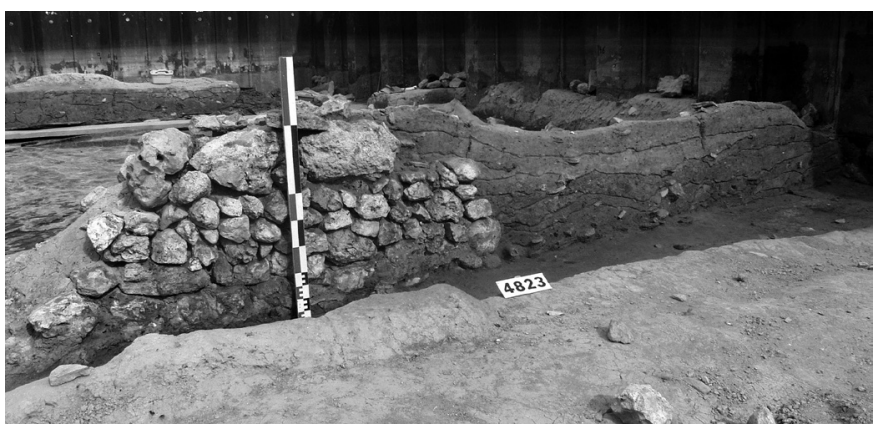

Fig. 68 - Combinaison de pierre et terre massive dans le mur MR51009 de la maison 130 de la zone 1 (photo : (C) UFRAL).

peuvent posséder un lit de pose fait de limon, qui sert à obtenir une surface horizontale préalablement à l'installation de l'élévation en terre.

\section{LES SOUBASSEMENTS ET MURS EN TERRE MASSIVE}

Les murs ou soubassements en terre massive ou bauge sont bâtis à base d'un mélange de terre et de végétaux ou d'autres fibres, qui peut aussi contenir des inclusions très diverses : des charbons de bois, des tessons, des os et des éléments provenant de la destruction d'autres murs (notamment des fragments de briques). Plusieurs possibilités de mise en œuvre du matériau sont attestées, mais on peut distinguer globalement deux modalités principales : des couches de terre litées superposées sur toute la hauteur du mur (fig. 68) ou des boules de terre projetées et postérieurement modelées (Chazelles, 1999, p. 229 ; Roux, 2003, p. 270 et Roux, infra, p. 107-109).

La technique de la terre massive sera largement employée sur le site de Lattara pendant tout le $\mathrm{V}^{\mathrm{e}} \mathrm{s}$. av. J.-C., où elle sera souvent utilisée en combinaison avec d'autres techniques (y compris d'autres formes de construction en terre). Dans la confection des murs, elle sera progressivement remplacée par des élévations en brique crue sur des solins en pierre au début du IV ${ }^{\mathrm{e}} \mathrm{s}$., et disparaîtra après le milieu de ce siècle. La terre massive continuera cependant à être employée pour l'élaboration de divers aménagements domestiques (banquettes ou autres).

Les largeurs des murs en terre massive sont comprises entre $0,55 \mathrm{~m}$ et $0,70 \mathrm{~m}$. Exceptionnellement, quelques murs dépassent ces dimensions : c'est le cas de ceux de la maison 132 de la zone 1 (450-425 av. J.-C.), dont les largeurs se situent entre $0,75 \mathrm{~m}$ et $0,90 \mathrm{~m}$ pour le mur nord et entre $1 \mathrm{~m}$ et 1,40 m pour le mur est (Belarte, Gailledrat, 2006). Quant aux élévations conservées, elles sont normalement peu

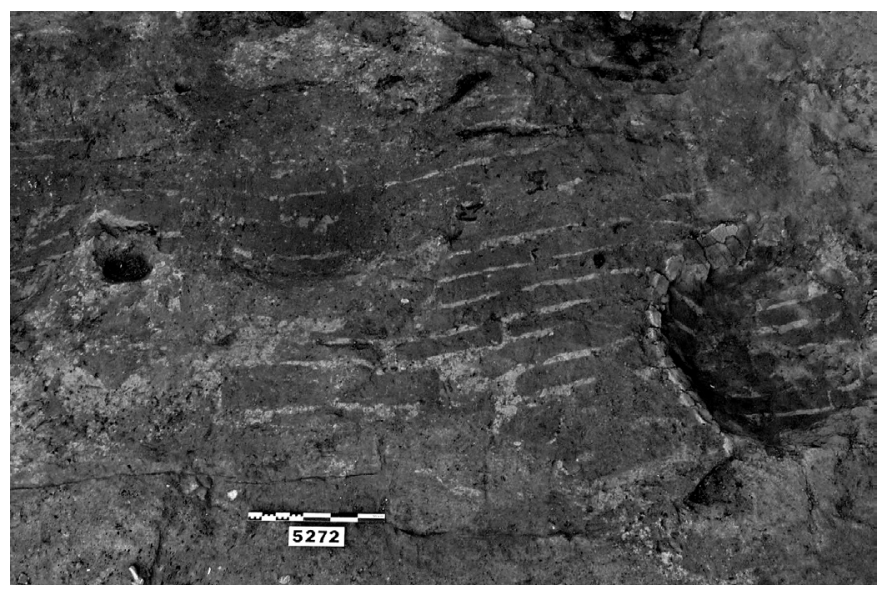

Fig. 69 - Vue du remblai 53027, formé par l'effondrement d'un mur de brique crue dans le secteur 49 de la zone 1 (vers 425 av. J.-C.) (photo : (C) UFRAL).

importantes, entre $10 \mathrm{~cm}$ et $45 \mathrm{~cm}$, avec quelques exceptions qui atteignent $90 \mathrm{~cm}$ de hauteur (Roux, 2003, p. 270).

\section{LES ÉLÉVATIONS EN BRIQUE GRUE}

L'adobe ou brique crue est attestée depuis les niveaux de fondation du site (fin du VI ${ }^{\mathrm{e}}$ s. av. J.-C.) dans la zone 27, en contexte culturel étrusque. Cette technique continuera à être employée lors des phases postérieures, jusqu'à la fin de l'occupation du site. La brique n'est jamais employée seule mais toujours sur des solins en pierre ou des soubassements en terre massive.

Les élévations en adobes sont parfois conservées en place, jusqu'à un maximum de 9 assises (dans le cas du mur MR50025 de la maison 122 de la zone 1, voir Roux, 1999, p. 22 fig. 14 et p. 55 fig. 68) : ces exemples permettent de mesurer les dimensions des briques et d'étudier leur mise en œuvre. Le plus souvent, les briques sont disposées en parpaing et boutisse et sont liées par une couche de limon de $1 \mathrm{~cm}$ à $3 \mathrm{~cm}$ d'épaisseur. Dans d'autres occasions, les briques sont identifiées dans des pans de murs effondrés vers l'intérieur des maisons. Dans ce cas, on ne peut pas connaître les dimensions totales des briques (seul les longueurs et les épaisseurs sont mesurables), mais l'on peut obtenir plus d'informations sur la hauteur originelle des élévations, qui parfois dépasse $3 \mathrm{~m}$. On prendra pour exemple le secteur 49 de la zone 1 qui, vers 425 av. J.-C., fut recouvert par une épaisse couche d'effondrement d'adobes (53027), sur $2 \mathrm{~m}$ à $3,40 \mathrm{~m}$ dans le sens est-ouest et près de $4 \mathrm{~m}$ dans le sens nord-sud (fig. 69) (Belarte, Gailledrat, 2006). 
Les briques conservées témoignent de l'utilisation de plusieurs modules, dont les plus courants sont de dimensions suivantes :

- 40-45 cm de long sur $35 \mathrm{~cm}$ de large, pour une épaisseur de $8-10 \mathrm{~cm}$,

- 30-34 cm de long sur 20-22 cm de large, pour une épaisseur de $7-8 \mathrm{~cm}$,

- 25-28 cm de long sur une largeur inconnue, pour une épaisseur de 7-8 $\mathrm{cm}$.

D'autres modules sont aussi attestés, comme les briques carrées (40 cm x $40 \mathrm{~cm}$ en moyenne) employées pour la construction de sols en adobes ou de banquettes.

\section{LES TOITURES}

Les toitures des maisons de Lattes du $\mathrm{V}^{\mathrm{e}}$ s. et du $\mathrm{IV}^{\mathrm{e}}$ s. av. J.-C. sont assez bien documentées, au moins en ce qui concerne les matériaux employés, grâce à plusieurs exemples de maisons incendiées dont la fouille a permis de récupérer une grande quantité d'informations.

Pour le $\mathrm{V}^{\mathrm{e}} \mathrm{s}$., nous disposons de la documentation livrée par la maison 130 de la zone 1, dont la destruction s'est produite dans le dernier quart du siècle. D'un côté et de l'autre d'un mur de refend, les sols des deux pièces (45A et 45B) qui composaient cette maison étaient recouverts de deux couches identiques de charbons de bois très denses, d'une épaisseur de $4 \mathrm{~cm}$ à $8 \mathrm{~cm}$ pour la pièce $45 \mathrm{~A}$ et de $2 \mathrm{~cm}$ à $4 \mathrm{~cm}$ pour la pièce $45 \mathrm{~B}$, provenant de la destruction de la toiture incendiée. Parmi les bois carbonisés, quelques éléments appartiennent vraisemblablement à des éléments de charpente. Les charbons de bois prélevés ont fait l'objet d'une étude préliminaire qui a permis de déterminer que la plupart appartenaient à de l'Arbousier (22 sur 23 déterminations parmi les charbons non fragmentés : $84 \%$ des charbons fragmentés); le Chêne et les Phragmites ont aussi été identifiés, mais en quantités bien inférieures (Champlot, 2003). Dans la pièce 45B, à proximité des murs ouest et sud, on a noté la présence d'éléments de planches brûlées, d'une épaisseur très faible $(0,50 \mathrm{~cm}$ en moyenne), qui comportent des petites pièces de bois de section ronde, de 1,50 cm de diamètre, ayant pu faire fonction de chevilles. D'autres éléments de section ronde (des branches de diamètre inférieur à $4 \mathrm{~cm}$ ) sont abondants à proximité des murs. Selon ces données, il est possible de restituer une couverture de Phragmites assemblés, placés sur une armature assez aérée.

Pour le IV ${ }^{\mathrm{e}}$ s. av. J.-C., la pièce XX de la maison 105 a livré des vestiges de toiture brûlée (charbons de bois, deux fragments de torchis avec empreintes d'armature) dans ses deux tiers orientaux. Parmi ces restes, les espèces les plus abondantes sont le Chêne vert, le Frêne, l'Orme et le Phragmite. Leur répartition en plan, très régulière, montre qu'il s'agit d'une seule structure effondrée dans l'ensemble de la pièce. D'autres essences peu abondantes sont peut-être intégrées dans l'architecture : ceps de Vigne de $2 \mathrm{~cm}$ à 2,50 $\mathrm{cm}$ de diamètre, Saule, Cornouiller. Enfin, des alignements de tiges de Pin d'Alep, Filaire et/ou Alaterne et de Rosacée Pomoïdée (de calibres minimums de $9 \mathrm{~cm}$ et $8 \mathrm{~cm}$ respectivement pour les deux dernières) font penser à des poutres ou chevrons disposés principalement selon la largeur de la maison (Buxó et al., 1996).

Dans les deux maisons, quelques fragments de torchis avec empreintes d'armature ont été repérés, mais ils ne sont pas suffisants pour penser à des couches de terre recouvrant toute la toiture.

En ce qui concerne l'inclinaison, et compte tenu de ce que nous venons d'indiquer sur les matériaux, nous exclurons a priori la possibilité de toitures plates. Les toitures à une seule pente semblent vraisemblables pour les maisons à une seule pièce et sans partitions intérieures ; dans la zone 1, la présence durant le $\mathrm{V}^{\mathrm{e}} \mathrm{s}$. av. J.-C. d'espaces de circulation internes au quartier fait penser que les toitures se déversaient vers ces ruelles servant également de drains. Dans quelques cas, l'existence de murs mitoyens suggère la possibilité de toitures à double pente.

\section{L'ACCÈS AUX MAISONS ET LA CIRCULATION}

Compte tenu de l'épierrement des solins en pierre et de l'abondance des murs en terre, l'identification des portes est souvent peu évidente, sauf lorsqu'il existe des seuils aménagés. Les portes identifiées ou restituées semblent indiquer que l'accès aux maisons s'effectuait à partir d'une rue passante ou d'une cour. Pour les quartiers où les maisons sont accolées au rempart, les ouvertures des portes sont orientées vers le côté opposé. Dans le cas de l'îlot 1, qui est perpendiculaire au rempart, les portes semblent orientées de préférence vers le nord (ouvrant sur la rue 101) mais des portes ouvrant sur les petites venelles entre les maisons sont également attestées durant le Ve s. av. J.-C. De toute manière, la faible largeur de ces venelles (de $50 \mathrm{~cm}$ à $80 \mathrm{~cm}$ ) indique que la circulation s'effectuait principale- 

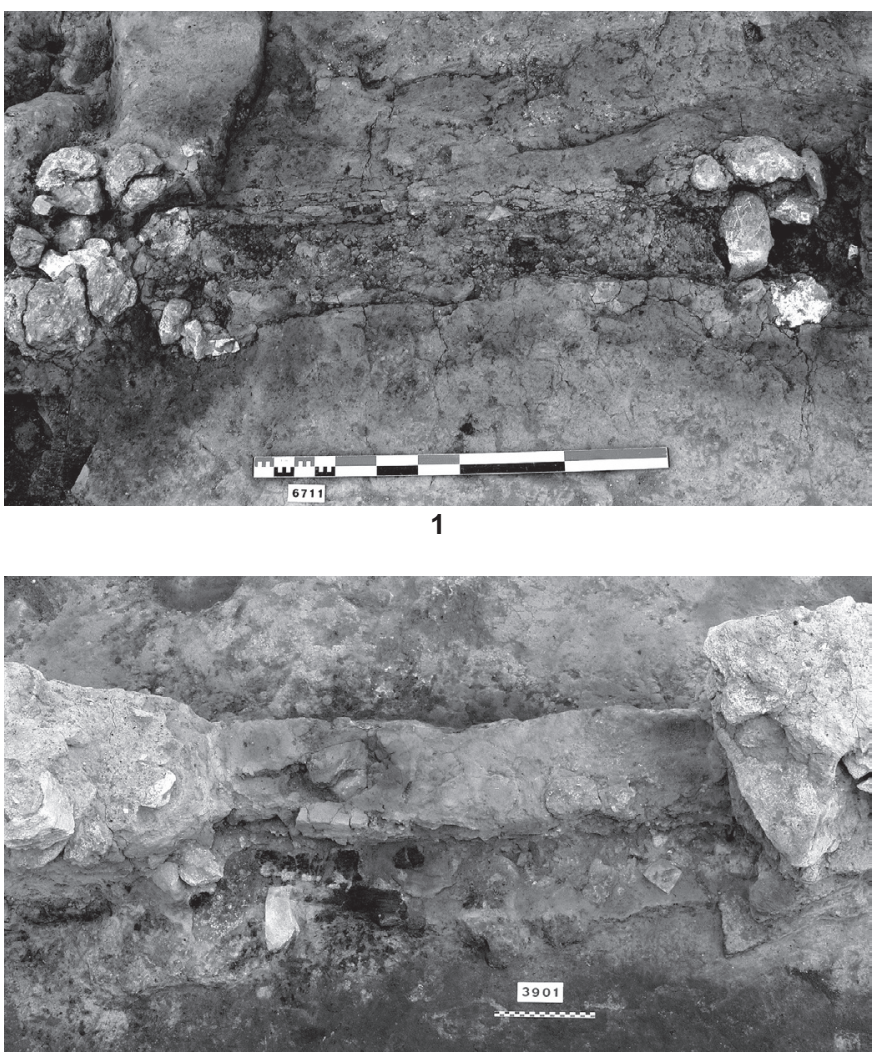

2

Fig. 70 - Accès aux maisons : 1, seuil en bois et crapaudines de la porte PR53262 de la maison 136 ; 2, seuil de la porte PR51089 de la maison 130, en moellons et cailloux recouverts d'enduit (photos : (C) UFRAL).

ment dans les rues les plus importantes. Souvent, l'accès à la maison se faisait à travers un appentis ou une cour qui ouvrait sur une rue.

Les portes identifiées présentent des largeurs variant entre $1 \mathrm{~m}$ et 1,50 m. Elles peuvent être centrées ou décalées dans la longueur du mur, mais rarement proches des angles. Dans quelques cas, les portes possèdent des seuils en terre maçonnée (porte PR1123 de la maison 103 ; porte PR51090 de la maison 130), en bois (porte PR53262 de la maison 136) (fig. 70, $\mathrm{n}^{\mathrm{o}} 1$ ) ou en moellons et cailloux recouverts d'enduit (porte PR51089 de la maison 130) (fig. 70, $n^{\circ} 2$ ). Les seuils peuvent être complétés par des alignements de tessons ou de briques. Enfin, des seuils aménagés à base de cailloux et de tessons sont également attestés. La porte PR53262 de la maison 136 (vers 450 av. J.-C.) (fig. 70, $\mathrm{n}^{\mathrm{o}} 1$ ) présente la particularité de posséder deux crapaudines faites d'un assemblage de pierres qui permettent de proposer une fermeture à double battant (Belarte, Gailledrat, 2006).

\section{LA FINITION ET L'ENTRETIEN DES MAISONS}

L'intérieur des maisons lattoises témoigne, pour toutes les périodes de la Protohistoire, d'un certain soin dans la finition des pièces, afin d'améliorer le confort de l'espace habitable. Les murs et les sols sont toujours enduits, le plus souvent d'une couche de limon (dont l'épaisseur peut varier de $1 \mathrm{~cm}$ à $3 \mathrm{~cm}$ ), qui peut également recouvrir des aménagements domestiques, tels que les banquettes.

$\mathrm{Au}$ cours des $\mathrm{V}^{\mathrm{e}}$ et $\mathrm{IV}^{\mathrm{e}} \mathrm{s}$. apparaissent des types de finition qui, dans quelques cas, perdureront jusqu'à la fin de la Protohistoire. C'est le cas des recharges de sable sur les sols, pratique attestée dans la maison 134 de la zone 1 (vers - 450) ou dans la pièce 45B de la maison 130 (vers -425), encore rares durant cette période et qui deviendront plus fréquentes entre le $\mathrm{III}^{\mathrm{e}} \mathrm{s}$. et le $\mathrm{I}^{\mathrm{er}} \mathrm{s}$. av. J.-C. (Belarte, 2004, p. 376). Les pavages en adobe, qui seront également caractéristiques du site jusque vers 150 av. J.-C., sont attestés pour la première fois vers - 400 dans la maison 102 (Roux, 1999, p. 71).

À l'intérieur des maisons, les sols apparaissent normalement propres, ce qui indique que des balayages réguliers avaient lieu. Les déchets se concentrent dans des fosses et surtout dans les zones extérieures qui, à l'époque en cause, jouent un rôle important en tant qu'espaces annexes des maisons. Des études de micromorphologie ont permis d'attester par ailleurs la présence de nattes sur les sols de certaines pièces (Cammas, 1999, p. 221).

\section{LES AMÉNAGEMENTS DOMESTIQUES}

Les aménagements domestiques sont assez fréquents dans les maisons $d u V^{e}$ s. et du $I^{e}$ s. (fig. 71). Les mieux attestées sont les structures liées à la cuisson (foyers et fours). Les foyers correspondent de manière majoritaire au type dit construit, avec sole établie le plus souvent sur un radier de tessons (en majorité des fragments d'amphore massaliète) installé dans une cuvette creusée dans le sol ; plus rarement, les radiers sont constitués de galets. Sur les radiers, les soles consistent normalement en une chape d'argile lissée. Les soles décorées, caractéristiques du Languedoc oriental, sont déjà attestées au $\mathrm{V}^{\mathrm{e}}$ s. av. J.-C. (fig. 72), mais ne deviennent fréquentes qu'au IV $^{\mathrm{e}}$ s. où elles présentent une grande diversité de décors (Roux, Raux, 1996), tandis qu'elles disparaissent au début du III $^{\mathrm{e}}$ s. av. J.-C. Les foyers lenticulaires ou à plat sont également présents, mais ils sont moins nombreux, sont 


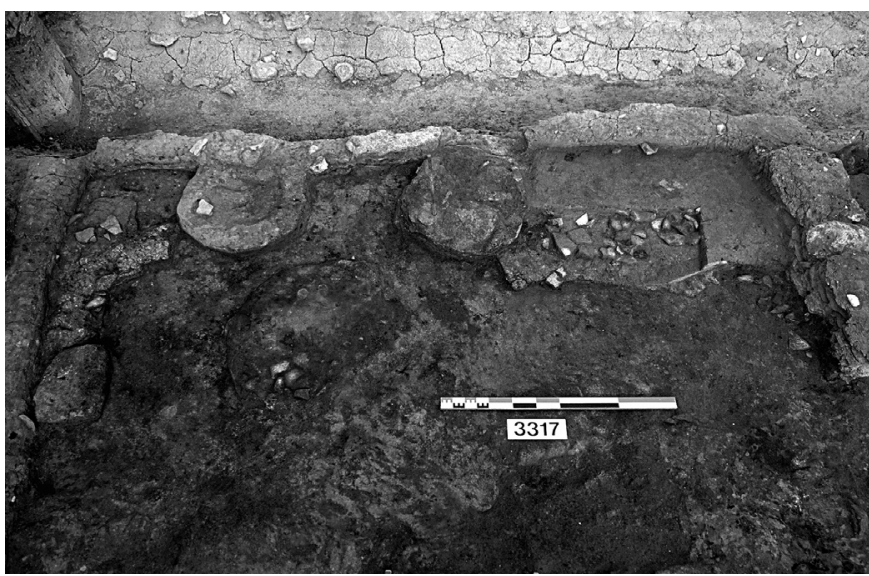

Fig. 71 - Aménagements domestiques contre le mur oriental de la pièce 39A de la maison 125 (425-400 av. J.-C.) : banquette BQ50294, support de vase SB50288, foyer FY50239 et cuve en torchis CV50289 (photo : (C) UFRAL).

normalement provisoires et se situent le plus fréquemment dans les cours. Enfin, on trouve quelques rares exemples de fosses-foyers au IV ${ }^{\mathrm{e}} \mathrm{s}$. av. J.-C. (en particulier dans la maison 129 de la zone 1), forme qui ne deviendra courante qu'à partir du III ${ }^{\mathrm{e}}$ s. av. J.-C.

Quant aux fours, ils sont en général situés dans des espaces ouverts (auvents et cours) et souvent protégés par des structures légères (fig. 73). La plupart des exemplaires sont de type fixe à sole pleine ; cependant des fours à cloche mobile sont aussi attestés. Dans leur majorité, ces fours sont formés d'un radier de tessons ou de galets recouvert par une sole d'argile, elle-même entourée d'une couronne de terre modelée de $6 \mathrm{~cm}$ à $7 \mathrm{~cm}$ d'épaisseur. L'élévation est rarement conservée sur plus de $10 \mathrm{~cm}$, mais elle est parfois effondrée à l'intérieur. Le four FR53169, dans l'espace ouvert 49 de la zone 1, présente une ouverture, visible sur un des côtés (fig. 74). Ces structures avaient une utilisation domestique : elles étaient vraisemblablement destinées à la cuisson de pain ou de galettes. Elles sont relativement nombreuses, ce qui laisse à penser que l'élaboration du pain n'était pas à cette époque une activité spécialisée, mais une tâche assumée par chaque famille.

Un deuxième groupe d'aménagements domestiques est formé par les banquettes, constructions basses adossées à un ou plusieurs murs d'une pièce, normalement faites de terre (en bauge ou en briques), bien que les banquettes en pierre soient également connues. Elles pouvaient être destinées au repos mais aussi au rangement de vases ou d'objets divers. Dans la plupart des cas, ces structures apparaissent dans les pièces qui contiennent des foyers, ce qui

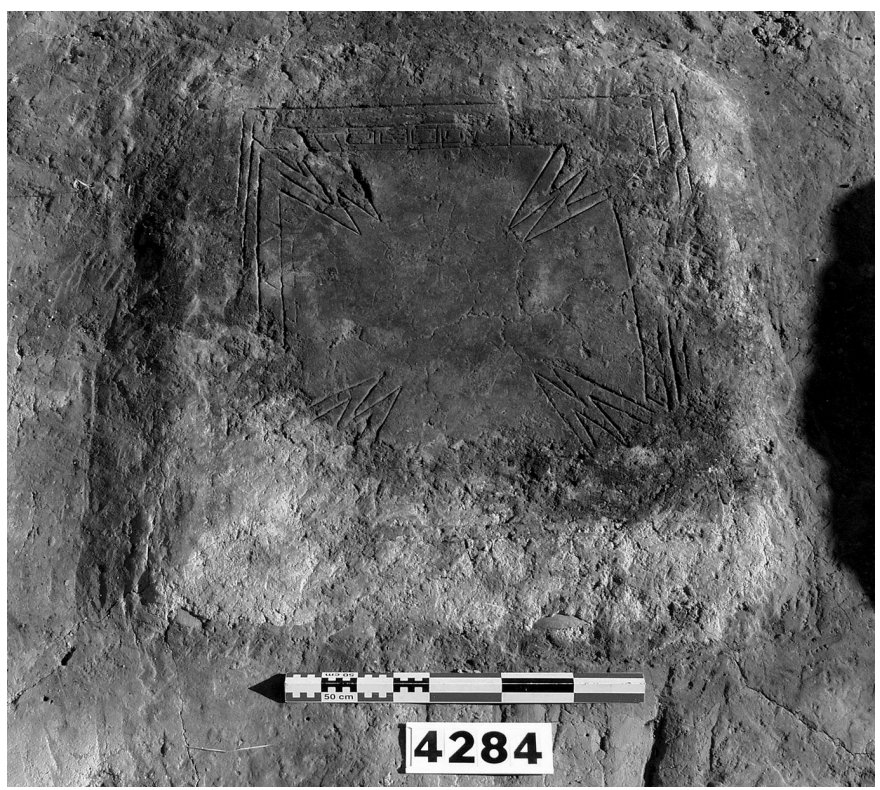

Fig. 72 - Sole décorée du foyer FY50371 du secteur 41 de la zone 1 (maison 126, dernier quart du Ve s. av. J.-C.) (photo : (C) UFRAL).

fait penser à des aménagements destinés aux repas. D’après les résultats des analyses de microtraces effectuées dans la zone 27 , la concentration de restes de faune aux pieds d'une banquette dans le secteur 5 permettrait d'associer cet aménagement avec la préparation ou la consommation de nourriture (Saffiotti, 1999, p. 208).

Dans plusieurs pièces de la zone 1 sont présentes au $\mathrm{V}^{\mathrm{e}} \mathrm{s}$. des structures bâties en pierre, à plan carré, que l'on désigne normalement par le terme de "tables basses » ou surfaces de travail et qui ne sont adossées à aucun mur. Il s'agit probablement de structures liées à la préparation des repas ou à d'autres activités domestiques ou artisanales, telles la mouture, le broyage, le travail des peaux, etc.

On trouve enfin, plus rarement, des aménagements liés au stockage (fosses destinées à implanter un vase, structures bâties, cuves en torchis). Les fosses d'implantation de vases sont très rares avant le $\mathrm{III}^{\mathrm{e}} \mathrm{s}$. av. J.-C. ; néanmoins, deux fosses ayant cette destination sont attestées dans la zone 1 durant le $\mathrm{V}^{\mathrm{e}} \mathrm{s}$. Les cuves en torchis sont normalement en rapport avec le stockage de graines ; dans la zone 1 , trois cuves sont attestées pour le $\mathrm{V}^{\mathrm{e}} \mathrm{s}$. et huit pour le IV ${ }^{\mathrm{e}}$ s. (fig. 71).

Des espaces vides d'aménagements, avec très peu de traces d'activités, sont aussi perceptibles, mais plus rarement. Ils étaient sans doute voués au repos ou peut-être au stockage. 


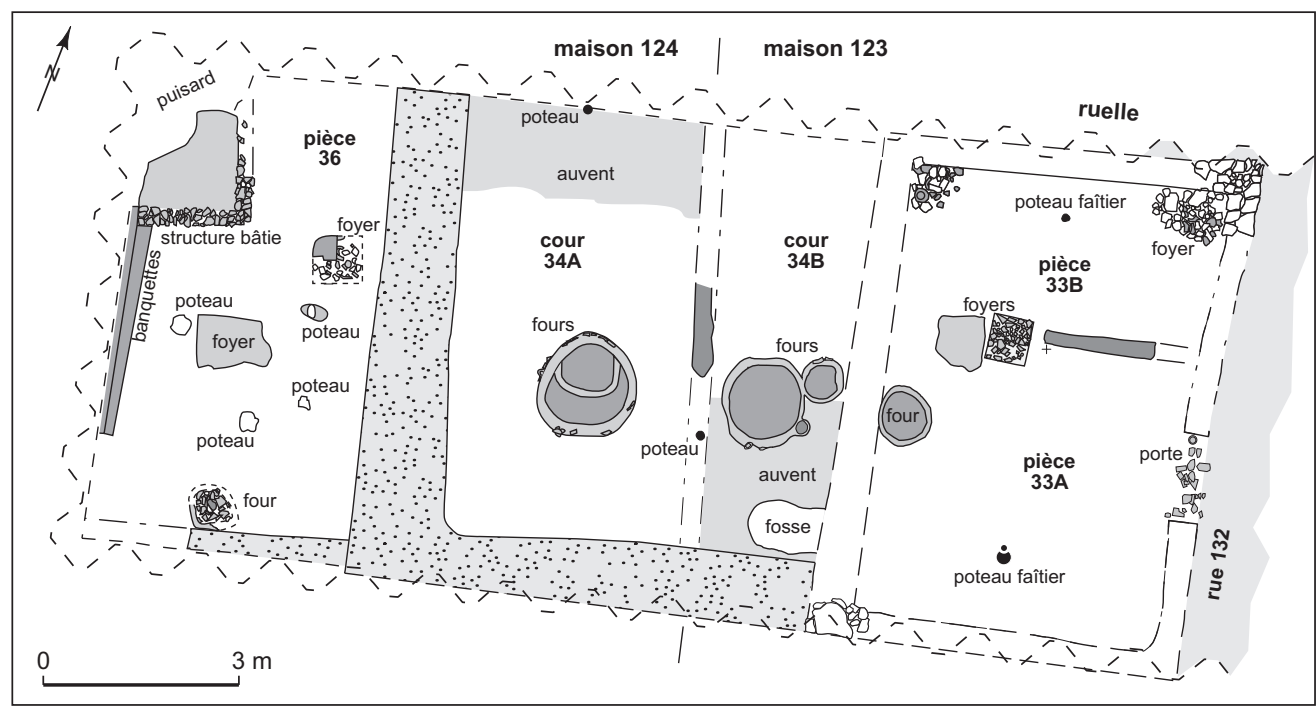

Fig. 73 - Plan des maisons 123 et 124 de la zone 1, avec la cour partagée 34 (DAO : (C) UFRAL).

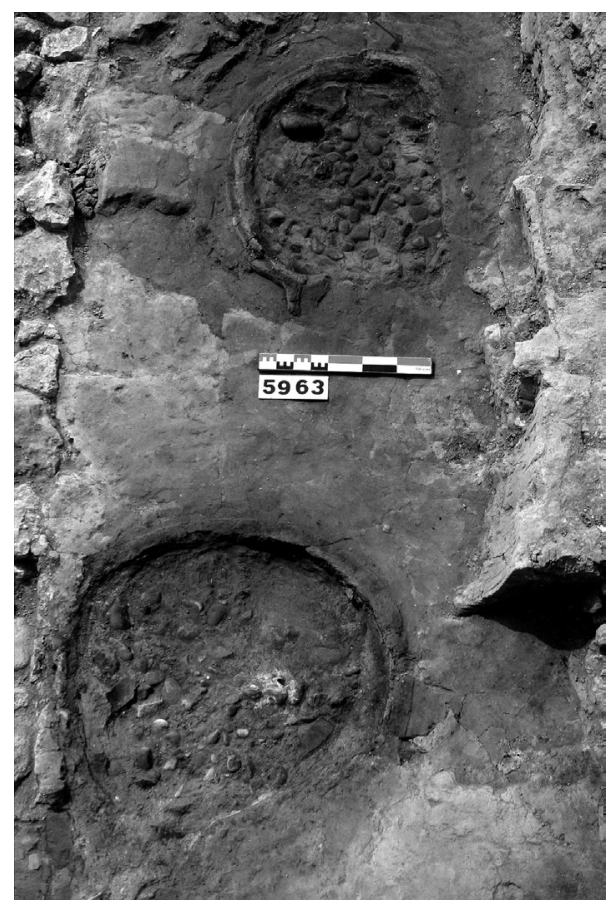

Fig. 74 - Fours FR53169 et FR53171 dans l'espace ouvert 49 de la zone 1 (vers 450-425 av. J.-C.) (photo : (C) UFRAL).

\section{PLANS ET SUPERFICIES DES MAISONS}

Depuis les niveaux de fondation de la ville et au cours des $\mathrm{V}^{\mathrm{e}} \mathrm{s}$. et $\mathrm{IV}^{\mathrm{e}} \mathrm{s}$. av. J.-C., une certaine diversité règne dans les plans des maisons et leurs superficies. La plupart des habitations reconnues possèdent deux espaces, mais les maisons à une pièce sont également présentes. Des unités domestiques à trois pièces sont attestées durant le $\mathrm{IV}^{\mathrm{e}} \mathrm{s}$., mais il n'est pas exclu qu'elles existaient antérieurement. Cependant les données disponibles sont souvent fragmentaires, du fait qu'elles proviennent de fouilles stratigraphiques sous forme de sondages et non de décapages en extension. Ainsi, pour plusieurs maisons, le nombre de pièces qui a pu être exploré ne correspond pas forcément au nombre total d'espaces qu'elles possédaient : c'est notamment le cas des différentes habitations qui se superposent à l'extrémité est de l'îlot 1 , accolé au rempart, et de quelques exemplaires de la zone 27. De même, la superficie totale des maisons n'est pas connue dans tous les cas. Un autre élément qui rend difficile l'interprétation de l'organisation domestique est le rapport étroit qui, durant la période concernée, semble avoir existé entre les espaces bâtis et les espaces extérieurs. Durant certaines phases d'occupation, les maisons à une ou deux pièces sont séparées par des secteurs non couverts ou des cours (fig. 64b) qui ont pu être utilisés de manière communautaire tout aussi bien que privative, voire être partagés par deux maisons attenantes. Ces difficultés expliquent que le calcul du nombre d'espaces composant réellement une maison et l'évaluation de sa superficie sont souvent approximatifs.

Au cours des différentes phases attestées dans la période prise en compte, nous disposons d'informations sur 49 maisons, dont 32 correspondent au IV ${ }^{\mathrm{e}} \mathrm{s}$. et $17 \mathrm{au} \mathrm{V}^{\mathrm{e}} \mathrm{s}$. Les données sur le IV $\mathrm{s}$. proviennent des zones 1, 4-sud, 7-est, 22, 24, 25, 27, 32 et 33. Pour le $\mathrm{V}^{\mathrm{e}} \mathrm{s}$., les données proviennent exclusivement des zones 1 et 27 . 


\section{TYPOLOGIE DES MAISONS DU V' S. AV. J.-C.}

Les maisons analysées pour cette époque correspondent à deux types différents selon le nombre de pièces : 8 maisons possèdent une seule pièce, sans cloison ou partition intérieure, et 9 maisons présentent deux pièces ; certaines maisons rangées parmi le type à une pièce ne sont cependant pas forcément complètes (fig. 75a).

$\mathrm{Si}$ nous classons ces maisons selon la disposition des pièces qui les composent et le rapport entre espaces couverts et non couverts, et tenant compte de la typologie établie par M. Py (Py, 1996a), nous pouvons retenir trois types : les 8 maisons à une seule pièce correspondent au type 1 ; les maisons à deux pièces se répartissent dans deux variantes : 6 maisons possèdent une pièce fermée précédée d'une cour et correspondent au type 2A de M. Py ; 3 maisons possèdent deux pièces fermées et appartiennent au type 2B (fig. 75b).

Au-delà de cette typologie formelle, il faut insister sur l'importance des espaces non couverts : parmi les 17 maisons analysées, 11 sont complètement couvertes et 6 possèdent des cours ou des espaces non couverts. On ajoutera à celles-ci les espaces ouverts 38/40 et 49 de la zone 1 qui, vers 400 av. J.-C., ont pu fonctionner comme des espaces annexes aux maisons attenantes.

En ce qui concerne la répartition des types de maisons par zone, les 8 exemplaires du type 1 ainsi que les 3 maisons du type 2B (c'est-à-dire à une ou deux pièces avec toute la superficie utile couverte) appartiennent à la zone 1. En revanche, les maisons du type $2 \mathrm{~A}$, avec deux pièces dont une (la partie avant) est une cour, sont attestées dans les zones 1 (3 maisons) et 27 (3 maisons).

Les données existantes font ressortir le peu de variété dans les types d'unités domestiques ou fonctionnelles, ainsi qu'une prédominance des maisons à deux cellules. D'autre part, si dans la zone 27 toutes les maisons analysées appartiennent à un même type, la zone 1 présente apparemment davantage de diversité. Ces différences, dans la mesure où l'on peut en juger sur la base d'une documentation encore fragmentaire, semblent tenir à la manière de répartir l'espace dans chaque quartier, c'est-à-dire principalement à l'environnement urbanistique.

Les données sont également partielles en ce qui concerne les superficies. Les informations disponibles montrent que, tous types confondus, la surface des maisons de cette époque se situe entre $23 \mathrm{~m}^{2}$ et $70 \mathrm{~m}^{2}$ hors tout, avec une moyenne de l'ordre de $44 \mathrm{~m}^{2}$. Il existe bien sûr un rapport entre le nombre de pièces et la superficie totale des unités domestiques : la surface moyenne des maisons du type 1 est de $37 \mathrm{~m}^{2}$, celles du type $2 \mathrm{~A}$ dépassent $53 \mathrm{~m}^{2}$ et celle du type 2B est proche de $40 \mathrm{~m}^{2}$ (fig. 75e).

\section{TYPOLOGIE DES MAISONS DU IV ${ }^{\mathrm{e}} \mathrm{S}$. AV. J.-G.}

Pour le IV ${ }^{\mathrm{e}}$ s., les informations sont un peu plus abondantes et les données reflètent une plus grande diversité dans les types de maisons ainsi qu'une légère augmentation des superficies. En effet, plus aucune maison à une pièce n'est attestée dans les zones explorées ; sur les 32 unités domestiques analysées, 16 possèdent deux pièces, 15 trois pièces et une seule comprend quatre pièces (fig. 75c).

Concernant la typologie, 5 maisons appartiennent au type 2A (pièce précédée d'une cour), 10 maisons correspondent au type 2B (deux pièces couvertes et disposées en enfilade), 1 maison appartient au type $2 \mathrm{C}$ (deux pièces couvertes, l'une à côté de l'autre), 6 maisons sont du type $3 \mathrm{~A}$ (deux pièces couvertes précédées d'une cour), 5 maisons correspondent au type 3B (deux pièces couvertes précédées d'une troisième pièce également couverte), 4 maisons appartiennent au type 3C (deux pièces disposées en angle entourant une cour ou un vestibule) et une maison appartient au type $4 \mathrm{~A}$ (quatre pièces séparées par des cloisons) (fig. $75 \mathrm{~d}$ ).

Il ne semble pas exister un rapport entre les différents types de maisons attestées durant le $\mathrm{IV}^{\mathrm{e}} \mathrm{s}$. et leur répartition dans l'espace : dans la plupart des zones pour lesquelles nous disposons d'informations pour cette période, des maisons à deux ou trois pièces sont également attestées.

Par ailleurs on observe une augmentation de la superficie totale des maisons, qui, durant le $\mathrm{IV}^{\mathrm{e}} \mathrm{s}$., tous types et phases confondus, est en moyenne de $62 \mathrm{~m}^{2}$, avec un minimum attesté de $28 \mathrm{~m}^{2}$ et un maximum de $110 \mathrm{~m}^{2}$. Nous devons signaler que la moyenne des superficies des maisons à trois pièces $\left(72 \mathrm{~m}^{2}\right)$ est nettement supérieure à celles des maisons à deux pièces $\left(48 \mathrm{~m}^{2}\right)$, tandis que la seule maison à quatre pièces est logiquement la plus grande $\left(110 \mathrm{~m}^{2}\right)$ (fig. $75 \mathrm{~d}, \mathrm{f}$ et g).

\section{LE RAPPORT ENTRE LES ESPACES COUVERTS ET NON COUVERTS}

Un élément remarquable est l'importance des cours ou espaces non couverts, aussi bien au $\mathrm{V}^{\mathrm{e}} \mathrm{s}$. qu'au IV $\mathrm{IV}^{\mathrm{e}} \mathrm{s}$. av. J.-C. Durant toutes les phases, les cours semblent avoir été utilisées en prédominance pour des activités culinaires, 


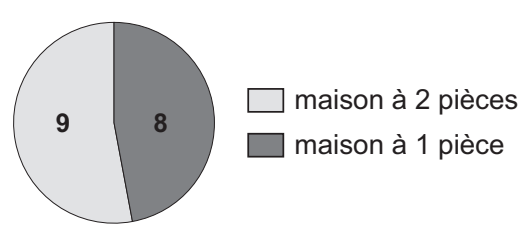

a

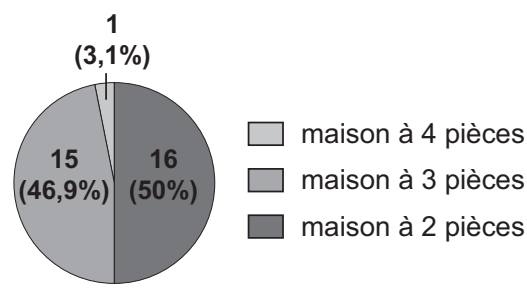

C

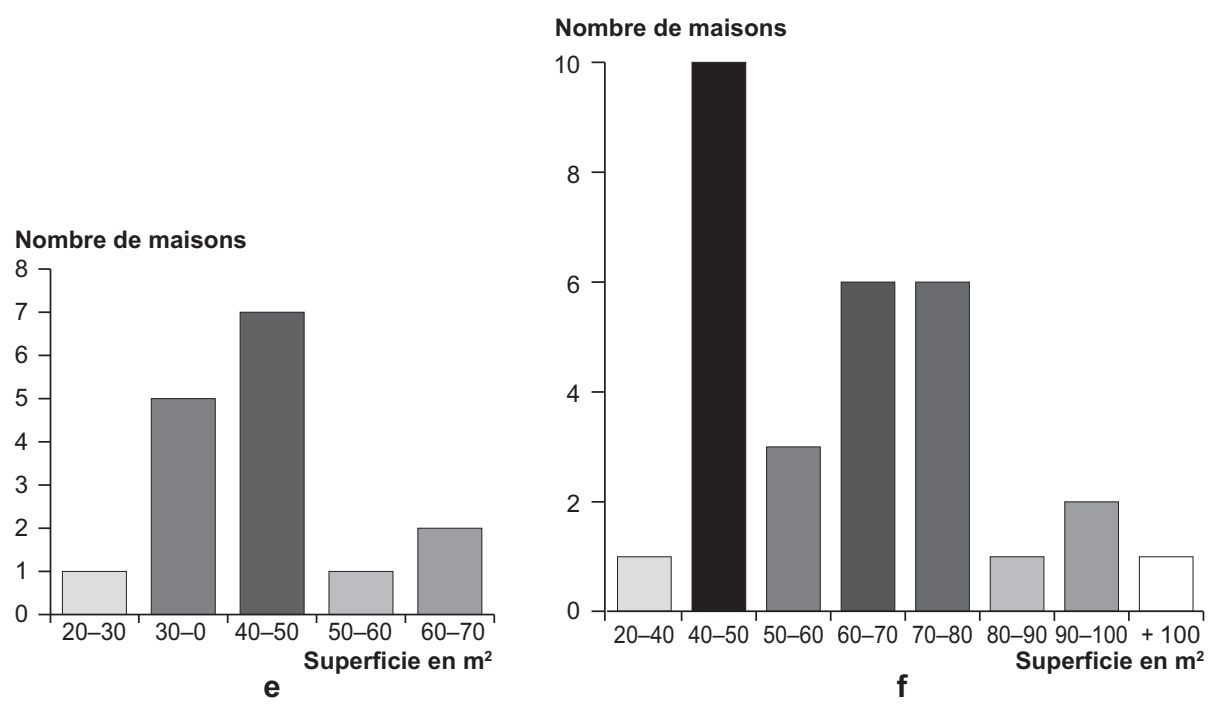

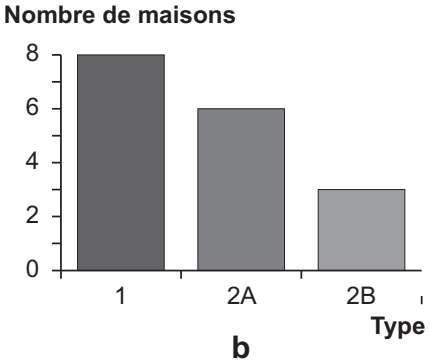

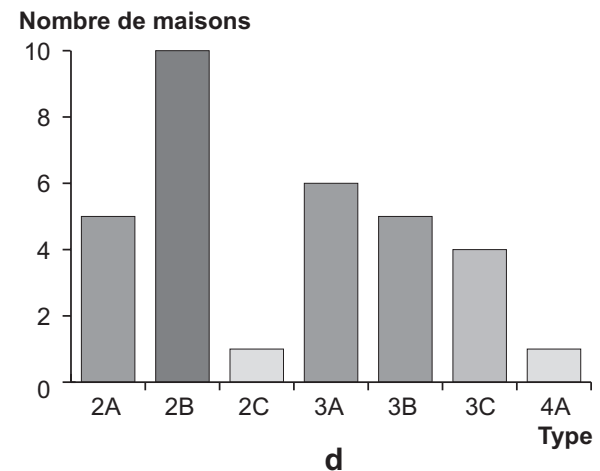

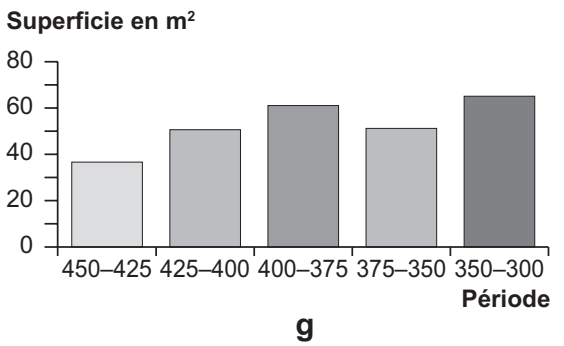

Fig. 75 - Données statistiques sur les maisons de Lattes datées des $V^{e}-I V^{e}$ s. av. J.-C. : a, rapport entre les maisons à une seule pièce et les maisons à deux pièces au $V^{e} s$.; $\boldsymbol{b}$, répartition des types $1,2 A$ et $2 B$ au $V^{e} s$. ; c, rapport entre les maisons à deux, trois et quatre pièces au IVes. ; $\boldsymbol{d}$, répartition des types 2, 3 et 4 au $I V^{e} s$, ; e, superficies des maisons du $V^{e} s$. ; $f$, superficies des maisons du IV s. ; g, évolution des superficies moyennes des maisons entre 450 et 300 av. J.-C. (DAO : M.-C. Belarte, ICREA/ICAC).

attestées par la présence de foyers et de fours (fig. 73 et 74), ainsi que de déchets provenant de la préparation des repas. La concentration de foyers dans quelques espaces ouverts, parfois associés à des fosses (secteur 49 de la zone 1 vers 425 av. J.-C.), pourrait indiquer que ce type d'espace était aussi voué à des activités artisanales. Même s'il est clair qu'une partie de ces secteurs ouverts était privatisée, on ne peut exclure pour d'autres une utilisation par les habitants de plusieurs maisons. Par exemple, une maie de pressoir destinée au pressurage des olives installée dans la cour 9 de la zone 27 pouvait avoir une utilisation collective. 
DE LA TYPOLOGIE

\section{À LA FONCTIONNALITÉ : L'UTILISATION DE L'ESPACE}

Il est possible de déterminer la fonction des espaces à partir des aménagements domestiques, du mobilier céramique et des objets récupérés dans chaque partie de chaque maison. Durant les périodes qui nous concernent, les aménagements domestiques visibles sont beaucoup plus abondants qu'ils ne le seront durant les étapes postérieures. On dispose donc de nombreux éléments pour attribuer des fonctions aux différentes pièces.

Les activités les plus nettement identifiées (et donc les plus souvent attestées) sont, durant toutes les périodes, celles en rapport avec la préparation et la consommation des repas : aménagements liés à la cuisson - foyers et fours -, restes de faune, céramiques de cuisine et vaisselle, instruments de mouture et de broyage, etc. En deuxième rang, en terme de nombre de traces se trouve le stockage, toujours à très petite échelle à cette époque. Des espaces livrant peu d'objets et peu de traces étaient peut-être réservés au repos (même si cette activité reste matériellement difficile à localiser).

Plus rarement, certains espaces étaient voués à une activité spécialisée, soit artisanale, soit vivrière (transformation d'aliments). La présence de plusieurs foyers, parmi lesquels une fosse-foyer cruciforme (fig. $76, \mathrm{n}^{\circ} 1$ ), laisse penser à une possible activité métallurgique dans la maison 129 durant le dernier quart du V ${ }^{\mathrm{e}}$ s. av. J.-C. (Belarte, Roux, 2003 , p. 78). Une maie de pressoir de la fin du V $\mathrm{V}^{\mathrm{e}} \mathrm{s}$. a été retrouvée en position fonctionnelle dans la cour 9 de la zone 27 : elle servait à la production d'huile et fut peut-être utilisée de manière communautaire (fig. $76, \mathrm{n}^{\mathrm{o}}$ 2). On doit noter enfin la trouvaille récente d'un possible tour de potier en bois dans la zone 1 (milieu du $\mathrm{V}^{\mathrm{e}} \mathrm{s}$.), qui indiquerait que la fabrication de céramiques avait lieu à l'intérieur d'une habitation (Chabal, 2006).

La répartition des activités est logiquement différente selon le nombre de pièces de chaque maison. Pour les unités domestiques à deux pièces, un des espaces est normalement voué à la cuisine et à la consommation des repas, l'autre au stockage. Dans le cas des maisons à trois pièces, la séparation se fait également entre cuisine et stockage, et le troisième espace sert à la consommation des repas ou au séjour/repos. On signalera enfin que les activités liées à la cuisine sont presque toujours attestées dans les pièces avant, plus proches d'un espace extérieur, et très souvent dans des espaces non couverts (auvents ou cours).
Les données mentionnées suggèrent donc que la maison $d u V^{e}$ s. et $d u I^{e}$ s. av. J.-C. à Lattes était un espace destiné principalement à la vie domestique, où avaient lieu la préparation et la consommation des repas, le repos des occupants ainsi que le stockage à petite échelle pour les besoins de la famille. On relève la rareté des espaces à caractère économique spécifique ; si plusieurs traces de petites productions domestiques sont confirmées, les indices d'artisanat spécialisé sont quasiment absents.

D'autres éléments suggèrent par ailleurs l'importance de la collaboration entre les familles dans certains domaines, comme la cuisson du pain ou la torréfaction de céréales : c'est le cas des espaces ouverts entre deux maisons (par exemple la cour partagée par les maisons 123 et 124 vers - 400, ainsi que probablement le secteur 49 vers - 450), qui étaient vraisemblablement utilisés par deux unités domestiques.

\section{DES RITUELS DOMESTIQUES}

Les pratiques rituelles associées à l'habitat, qui sont relativement fréquentes à Lattes durant toute l'occupation protohistorique, sont attestées depuis le dernier quart du $\mathrm{V}^{\mathrm{e}} \mathrm{s}$. et surtout durant le $\mathrm{IV}^{\mathrm{e}} \mathrm{s}$. av. J.-C. Il s'agit de sépultures d'enfants morts en bas âge, de dépôts votifs d'animaux (ovins, caprins, bœuf, serpent, chien) ou de dépôts de petits vases. Ils sont situés à l'intérieur des pièces ou dans des espaces ouverts étroitement associés à une maison ; ils peuvent être aussi en relation avec un élément architectural : mur, seuil ou banquette (Fabre, Gardeisen, 1999). Ces dépôts à caractère votif ou magique coïncident souvent avec le réaménagement des îlots, ce qui suggère l'existence de pratiques rituelles visant à la protection des bâtiments ou à leur purification lors d'une reconstruction. Ce type de pratique appartient à une tradition attestée durant la Protohistoire dans plusieurs régions de la Méditerranée, qui perdure jusqu'à la romanisation et même au-delà (Collectif, 1997).

$$
\text { * }
$$

Les informations livrées par les maisons des $\mathrm{V}^{\mathrm{e}} \mathrm{s}$. et IV $\mathrm{s}$. de Lattes constituent d'importants témoins du processus de mise en place de la structure urbaine dans une civilisation protohistorique. Elles participent, avec d'autres exemples régionaux, à un processus de stabilisation et de normalisation de l'habitat.

Les maisons découvertes dans les zones 1 et 27 , où les espaces ouverts (cours et appentis) occupent une place importante, présentent une certaine parenté avec des cas 


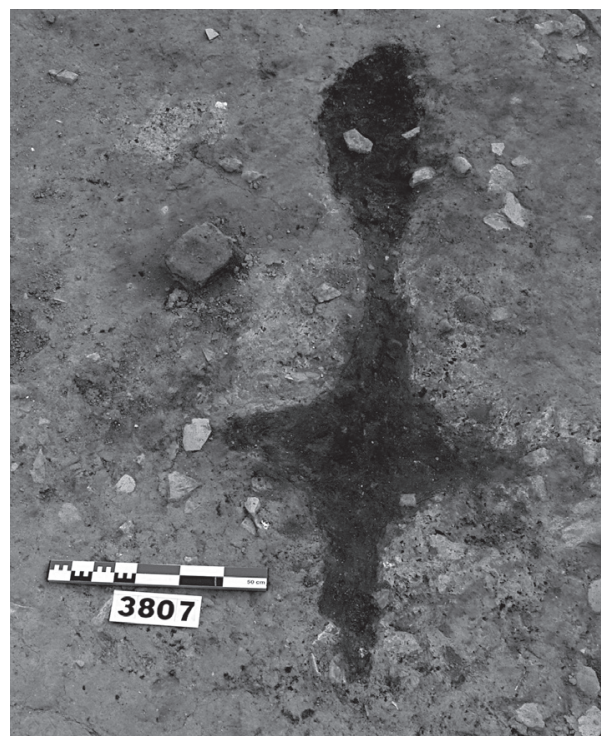

1

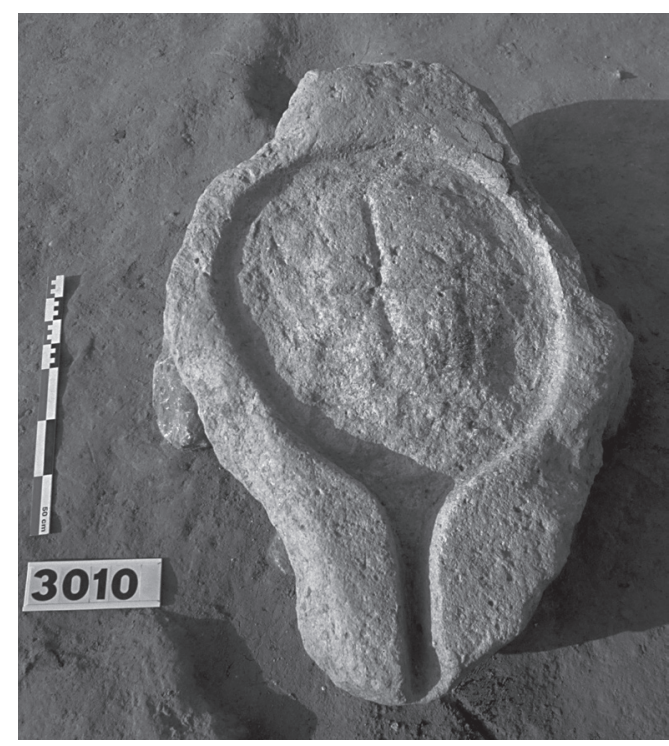

2

Fig. 76 - Témoins d'activités artisanales et vivrières dans les maisons ou les cours : 1, fosse-foyer FY50314 de la pièce 44 de la maison 129 de la zone 1 (vers 425-400); 2, maie de pressoir en position fonctionnelle dans la cour 9 de la zone 27 (vers 400 av. J.-C.) (photos : (C) UFRAL).

attestés sur des sites contemporains, tels que le Plan-dela-Tour à Gailhan, le Marduel à Saint-Bonnet-du-Gard ou Montlaurès à Narbonne.

Sur ce dernier site, autour de 500 av. J.-C., les maisons ne partagent pas de murs mitoyens mais sont séparées les unes des autres, tout en s'insérant dans des lignes directrices qui font penser à un lotissement prédéfini, avec attribution à chaque entité non seulement d'une habitation, mais aussi d'un espace ouvert voué aux activités culinaires (Chazelles, 2005, p. 248).

Sur le site du Marduel, une maison accolée au rempart, formée de deux pièces couvertes précédées par une grande cour enclose, est attestée dès la fin du VI ${ }^{\mathrm{e}}$ s. av. J.-C. (Py, Lebeaupin, 1992 et 1994).

En Roussillon, sur le site de Salses-le-Château (fondé au début $\mathrm{du} \mathrm{V}^{\mathrm{e}} \mathrm{s}$.) a été fouillé un quartier de maisons mitoyennes accolées au rempart ; il s'agit d'habitations à deux, trois ou quatre pièces, disposées en îlots réguliers, dont les plans sont assez proches de ceux des maisons de Lattes à partir de 425 av. J.-C. (Ugolini et al., 2000, p. 189, fig. 3).

Les principes mis en ouvre par les maisons et l'urbanisme de Lattes se retrouvent donc sur d'autres sites languedociens au sein de ce qui apparaît comme une période de tâtonnements ou d'expérimentations, où des espaces ouverts alternent avec des espaces couverts, des maisons en dur avec des maisons en torchis, des architectures indépendantes avec des bâtiments accolés.
L'analyse des plans des maisons suggère une certaine évolution au cours de ces deux siècles, qui comporterait un agrandissement des superficies, une augmentation du nombre des espaces à l'intérieur de l'unité domestique et une disparition des murs doublés et des venelles au profit d'îlots plus compacts faits de maisons mitoyennes. Peu après, on assistera à une réduction de l'importance des espaces ouverts par rapport aux espaces couverts. Si la superficie moyenne des maisons du $\mathrm{V}^{\mathrm{e}}$ s. est de $43 \mathrm{~m}^{2}$, celle-ci augmente jusqu'à $62 \mathrm{~m}^{2}$ durant le $\mathrm{IV}^{\mathrm{e}} \mathrm{s}$. Il est intéressant en ce sens de rappeler les dimensions du module théorique qui a été proposé pour les îlots du second âge $\mathrm{du}$ Fer de Lattes, qui tournerait autour de $64 \mathrm{~m}^{2}$ hors tout (Garcia, 1996, p. 20), superficie qui pourrait correspondre aux besoins d'une famille et à un lotissement du terrain. Ce module théorique a pu être formalisé vers 400 av. J.-C. à la suite des évolutions constatées durant les périodes antérieures.

L'analyse globale des maisons de Lattes ne permet pas d'identifier pour ces périodes anciennes de variations importantes dans les dimensions, les finitions ou les aménagements intérieurs qui inciteraient à conclure à une nette différenciation sociale entre les habitants des quartiers étudiés. En revanche, les dimensions observées sur ce site sont supérieures à la moyenne des superficies des maisons du second âge du Fer languedocien qui, d'après une synthèse récente (Dedet, 1999, p. 332), serait de $22 \mathrm{~m}^{2}$ 
vers 500 av. J.-C. et de $35 \mathrm{~m}^{2}$ vers 300 av. J.-C. De même les maisons à trois pièces couvertes, déjà attestées à Lattes au $\mathrm{IV}^{\mathrm{e}} \mathrm{s}$., semblent rares dans le contexte régional antérieurement au $\mathrm{II}^{\mathrm{e}}$ s. (Dedet, 1999, p. 331).

La surface des maisons ou la relative complexité de leur plan ne sont pas les seuls éléments qui permettent d'établir des différences entre Lattes et son environnement indigène : la superficie de la ville, la précocité et la monumentalité de son rempart, la régularité de son urbanisme, l'important volume des apports commerciaux durant toute la durée de son occupation, sont autant de points distinctifs ; l'architecture domestique du site, même si elle peut apparaître modeste, s'ajoute à ces aspects pour témoigner à sa façon du dynamisme de cette agglomération. 